International Journal of Distributed and Parallel Systems (IJDPS) Vol.1, No.2, November 2010

\title{
THE INTERACTIVE ZOOOZ GUIDE
}

\author{
Thang Nguyen and Hao Shi \\ School of Engineering and Science, Victoria University \\ Melbourne, Australia \\ johnedmcg.com.au and hao.shievu.edu.au
}

\begin{abstract}
GPS (Global Positioning System) technology is widely known for its ability to track down devices in real time. Combined with mobile phones, it has become a very powerful tool with a great potential for future development of mobile GPS applications. The Interactive ZooOz Guide was a final year industry project carried out by seven students from three separate courses to tackle a project that involves upgrading the Melbourne Zoo's mapping system through the use of GPS technology. The aim of the project is to explore the potential capability of using GPS in the Zoo environment. The proposed system uses a PDA device with a GPS receiver that tracks users' location in real time as they are touring around the Zoo. In this paper, an insight into GPS technology is briefly reviewed and the design and implementation of "Interactive ZooOZ Guide" is described. Then GUI (Graphical User Interface) is presented in detail. Finally conclusion is drawn from the "proof of concept" prototype.
\end{abstract}

\section{KEYWORDS}

Industry Projects, Global Positioning System (GPS), PDA (Personal Digital Assistant), Interactive Zoo Guide

\section{INTRODUCTION}

Global Positioning System is a satellite based technology where each satellite broadcasts a signal from space to be read by a GPS receiver to calculate the three-dimensional location (latitude, longitude and altitude) of a person carrying the receiver [1]. The transmitted data is normally translated to an electronic mapping system that regularly updates as the user moves.

GPS was originally designed for military application up until the early 1980s when a civilian airline strayed into prohibited airspace and consequently shot down killing all the passengers onboard. Since the incident, the U.S. government announced that GPS would be available for civilian use but restricted to a weaker signal. But in 2000, they quashed the restriction realizing the benefits for civilian use and gave way to a new era in GPS technology [1].

Soon after, mobile phone technology became 3rd generation, which meant the devices were able to handle vast amounts of data transmission and utilize features such as the Internet, digital camera and video conferencing. Since 2004, we were able to use GPS technology on mobile devices but it is still under development as it is only available to phones with a GPS receiver and more practical to phones with a larger screen [2].

\section{THE INTERACTIVE ZOOOZ GUIDE}

The aim of the Interactive $\mathrm{ZooOz}$ guide was to overcome the difficulties of navigating through Melbourne Zoo by using existing technology [3, 4]. It was developed as a "proof of concept" to explore the potential limitations of using GPS in such an environment.

The developed ZooOz system was programmed using C Sharp .NET with an external GPS library and was deployed to a Personal Digital Assistant (PDA). The system shown in Figure 1 
International Journal of Distributed and Parallel Systems (IJDPS) Vol.1, No.2, November 2010 begins with the PDA communicating with the GPS receiver assuming that it is on. The ZooOz application from the PDA sends a request query to the receiver in frequent intervals (via Bluetooth) while the receiver is constantly feeding on signal information sent out by GPS satellites. When the receiver reads the first pair of coordinates, it sends the information back to the application to be translated to decimal latitude and longitude values as illustrated in Figure 1.

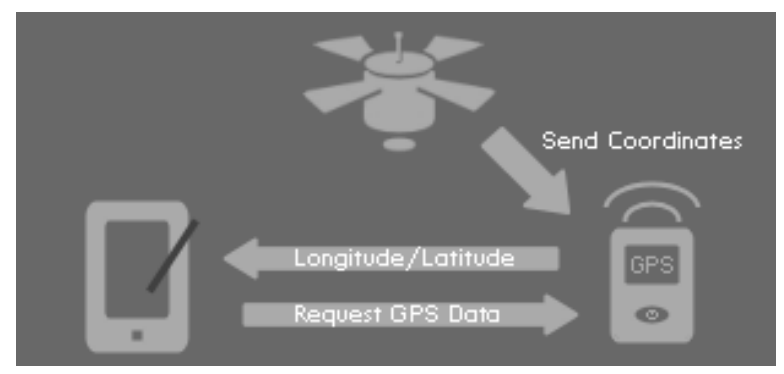

Figure 1. The Interactive ZooOz guide - Information Flow [3]

\section{SYSTEM OVERVIEW}

The system consists of three key components as illustrated in Figure 2:

- $\quad$ PDA device supporting the main program

- A GPS receiver

- A relational database

The GPS receiver is connected to the PDA via Bluetooth. The system allows its users to check their connectivity with the GPS receiver and then provides the current location, i.e. longitude and latitude of the PDA device. Once the main program is started the program activates the connection to the GPS Device. After the connection is established with the GPS satellites, it displays main menus from a relational database and executes the commands according to the users' requests.

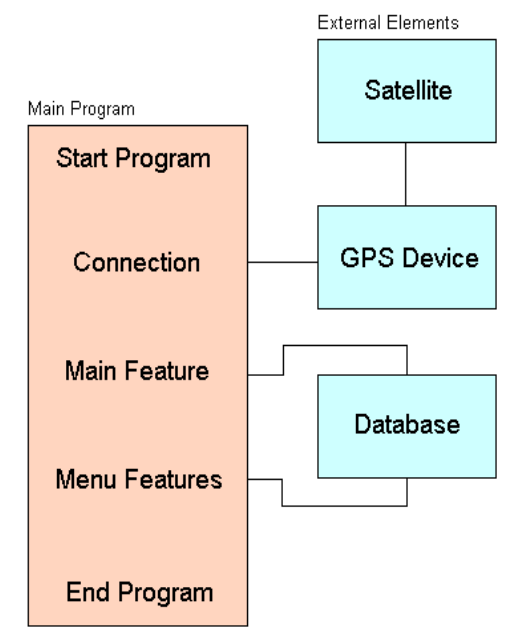

Figure 2. System overview [5] 
International Journal of Distributed and Parallel Systems (IJDPS) Vol.1, No.2, November 2010

The use case diagram below shows functions which users can interact with once the map interface is presented. The actor in Figure 3 is presented using a stick figure people and the functions are presented in an oval.

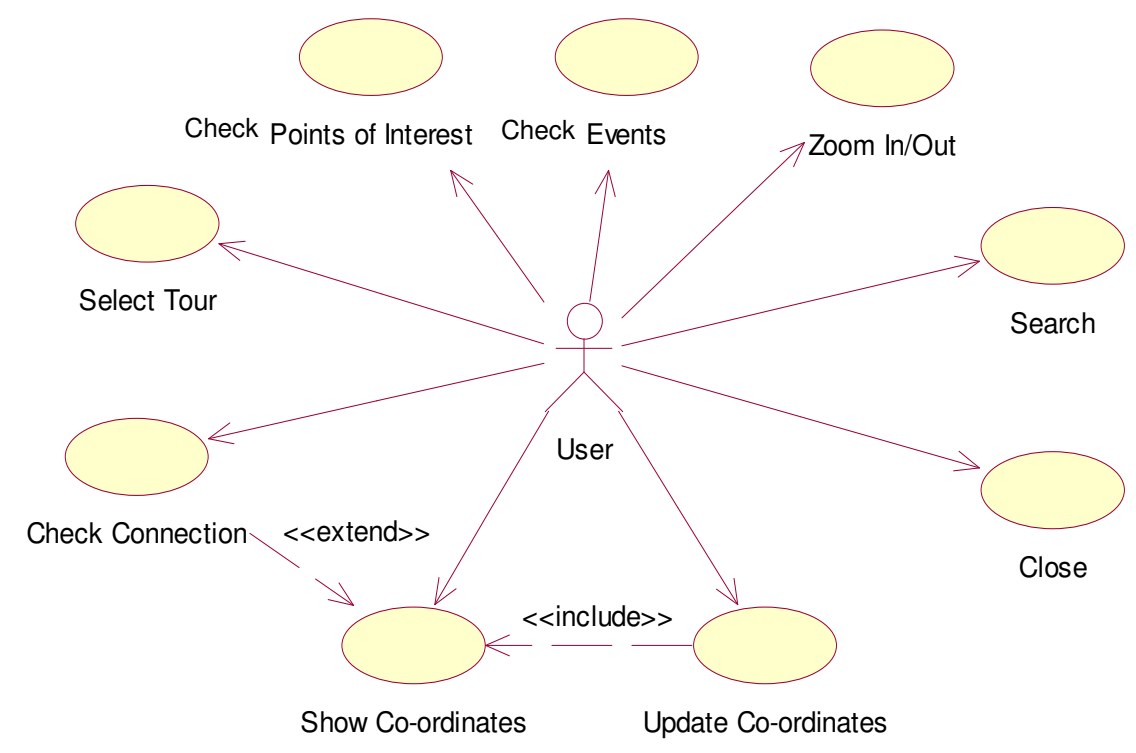

Figure 3. Use Case diagram [5]

\section{DESIGN AND IMPLEMENTATION}

Also some basic configuration between the PDA and GPS receiver must be set up. First is making sure that all devices are discoverable by the PDA by turning on the Bluetooth setting. Then through the connection settings a partnership must be made between the PDA and GPS receiver. The GPS receiver will connect to an open communication port specified by the PDA [6].

\subsection{Development Tools}

For testing and development, the following tools were used:

- Microsoft Visual C\# .Net

- External Dynamic Link Library (DLL) called Franson GPSGate

- ActiveSync (for Testing)

- PhotoShop (image manipulation)

- SprintDB Pro

\subsection{System Setup}

Prior to running, a few software packages have to be installed on the PDA for it to function.

- Windows Mobile $20032^{\text {nd }}$ Edition

- Macromedia Flash Player 7 for Pocket PC

- SprintDB Pro 3.1

- SprintDB Pro Desktop Companion 2.1 
International Journal of Distributed and Parallel Systems (IJDPS) Vol.1, No.2, November 2010

\subsection{Development}

The main program has two procedures. In the Form_Load procedure, the application tries to find the communication port holding the GPS receiver. Once it is found, it automatically connects to the receiver.

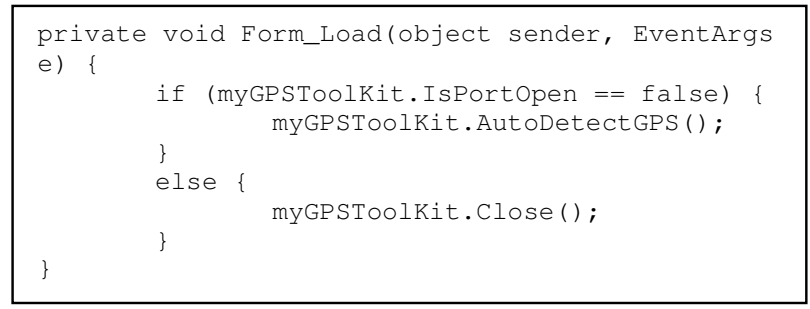

Figure 4. Code - Connecting to the GPS receiver [5]

The next procedure called GPSToolKit_PositionUpdate runs in an endless loop to simulate real time readings. In every loop, the application sends a request for information from the GPS receiver. When a valid position is read, the latitude and longitude are printed and then used to determine the position of the cursor on the map. If a valid position is not read, it will simply display a No Latitude/Longitude message but will remain connected to the GPS receiver.

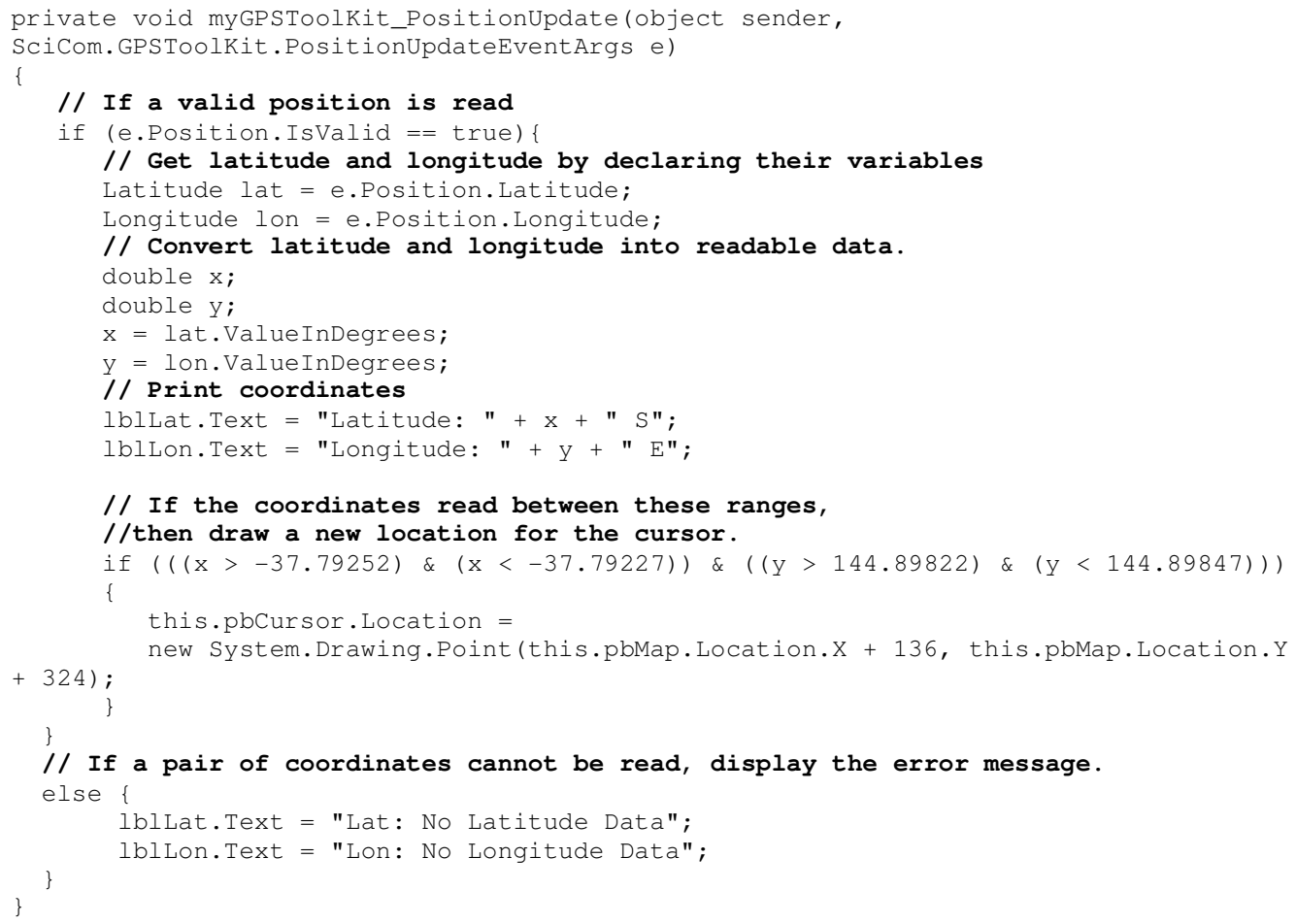

Figure 5. Code - Reading and updating coordinates [5]

When the coordinates are updated, they are checked if they fall within a range of 5 metres (coordinate metres $\approx 0.00025$ ) of a particular zone. If the condition is successful, the system gives a new location of the cursor image to be placed on the map. 


\section{GRAPHICAL USER INTERFACE DESIGN}

The ZooOz guide allows a user to interact with the system through the PDA's touch technology. With the touch screen users are able to browse through menus, view hot spots and play games. But the main features of the application are the interactive map and the points of interest (hot spots). The interactive map (Figure 4) is a regular JPEG image derived from a CAD (Computer Aided Design) image of the zoo. Layering on top of the map is a cursor that only moves when a range condition is satisfied.

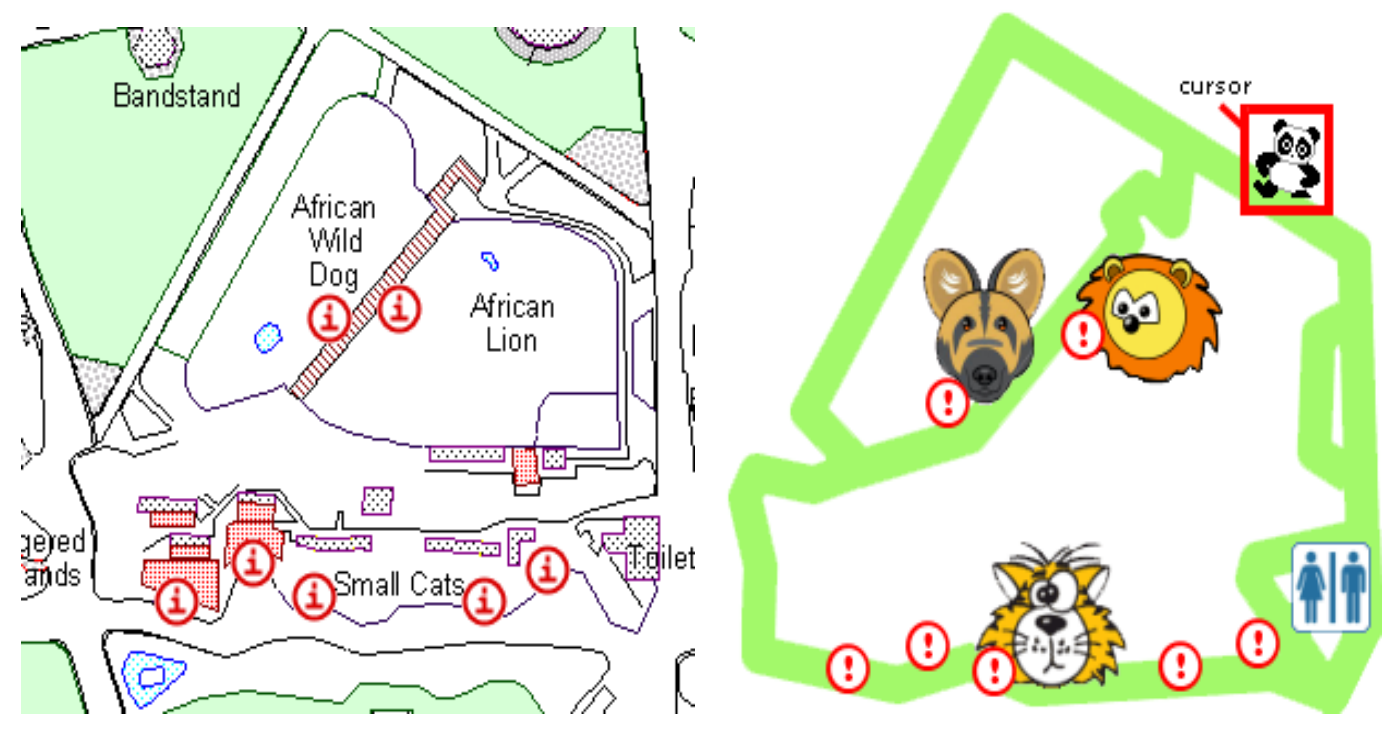

Figure 6. Zoo map [5]

A hot spot is activated when the cursor and itself meets. The red mark will animate to bring the user's attention to the hot spot. Each hot spot corresponds to a particular animal which will display information of that animal when pressed. Cursor change works in conjunction with GPS readings. At present, the cursor simply jumps from one spot to another using area zones that are triggered when the GPS coordinates fall within the range of those zones. To simulate constant movement, the cursor change in $\mathrm{X}$ and $\mathrm{Y}$ would have to be calculated with the change in longitude and latitude. Readings that had 5 or more decimal places were changing erratically when the GPS receiver was in a stationary position. That data was unusable for the application. But there was a consistent change of 0.0001 latitude/longitude unit (4-decimal points) for every 2 metres moved, which was used instead. Fifteen pairs of GPS data was collected and repeatedly tested within the zoo environment for consistency, but due to the small working area and interference by tall flora and weather, the results varied.

\subsection{Startup Screen}

The startup screen is incorporated the Melbourne Zoo's logo and features a leopard at the background to represent the 'Big Cats' as shown in Figure 7. Once the startup screen disappears, the program identifies the COM port number of the GPS Bluetooth device, establishes the communications with the GPS satellites via the GPS receiver as shown in Figure 8 , and displays the main screen. 
International Journal of Distributed and Parallel Systems (IJDPS) Vol.1, No.2, November 2010

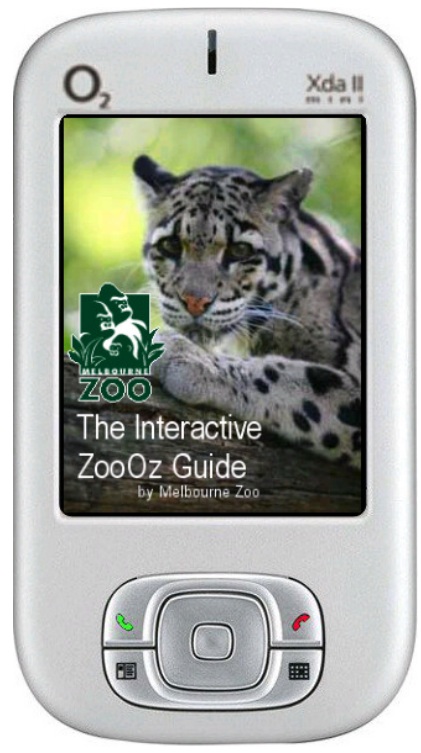

Figure 7. Startup screen [5]

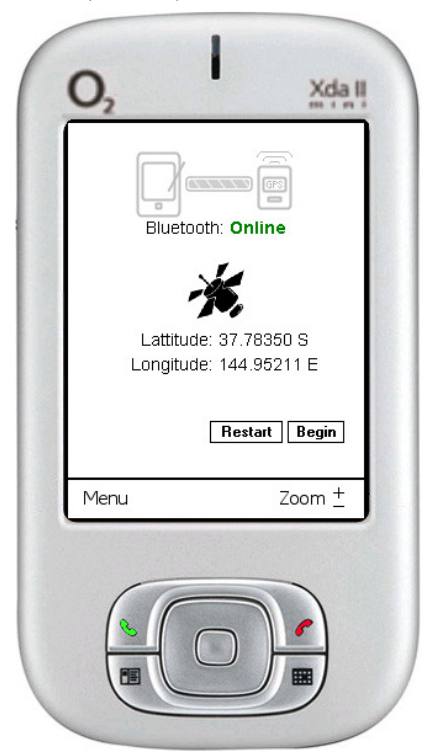

Figure 8. Connection screen [5]

\subsection{Menu Screen and Submenu Screens}

There are two functionalities available: Menu and Zoom, which can be seen from the main screen in Figure 9. Once the menu is clicked, it shows the six submenus as illustrated in Figure 10. Then the user can select one of the six options from Check Connection, Show Coordinates, Tour Guide, Search, Events (timetable) and Close.

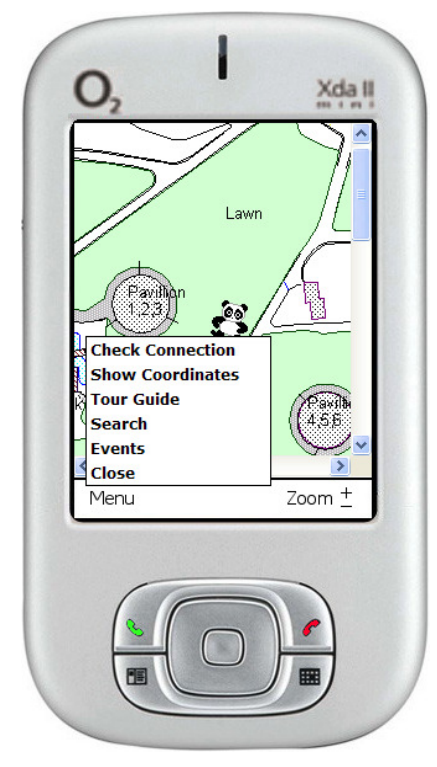

Figure 9. Menu screen [5]

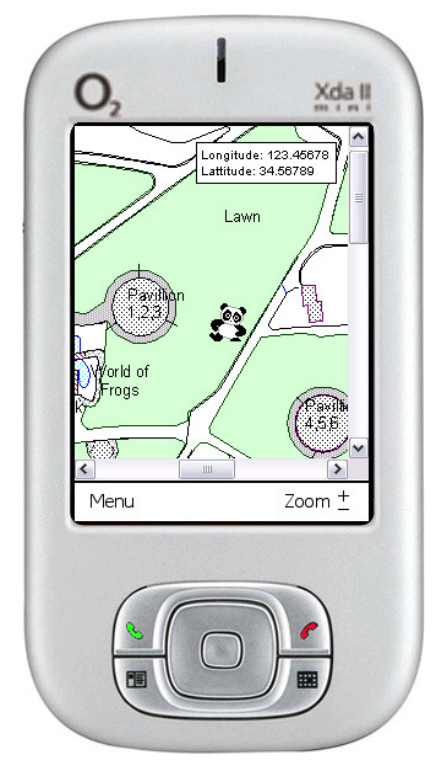

Figure 10. Coordinates screen [5]

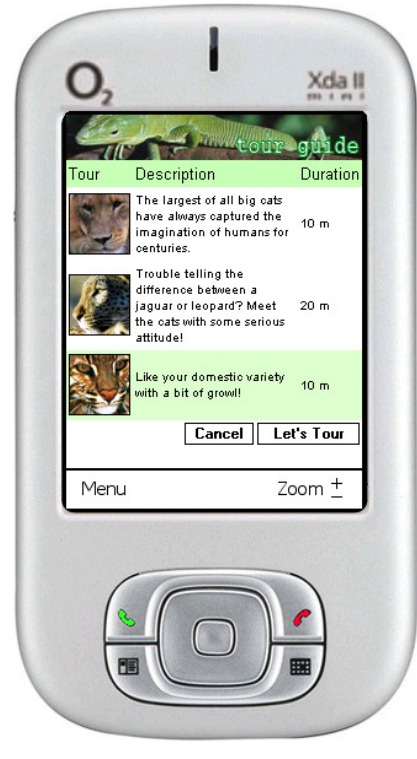

Figure 11. Tour guide screen

[5]

The first two submenus are associated with GPS configuration/setting and users can check GPS connection via the connection screen as shown in Figure 12 or sees the display of latitude and longitude on the screen as shown in Figure 13. The tour guide contents are created by the multimedia students. The user can select each individual tour as shown in Figure 14 and watch 
International Journal of Distributed and Parallel Systems (IJDPS) Vol.1, No.2, November 2010

the video and/or listen to the audio related to the tour. The tour map is developed by the computer science students. The track of each tour can be displayed on the screen to give the users a clear idea where the tour starts and ends. The search submenu is designed to provide its users the location information such as the distance and direction at the current position. This search facility allows the users to search for locations of importance within the Zoo, e.g. the closest toilet or the quickest way to get to the cafeteria. The events submenu as shown in Figure 11 offers the latest timetable for special activities on the day. The exit screen allows the users to exit the program after conformation as shown in Figure 14.

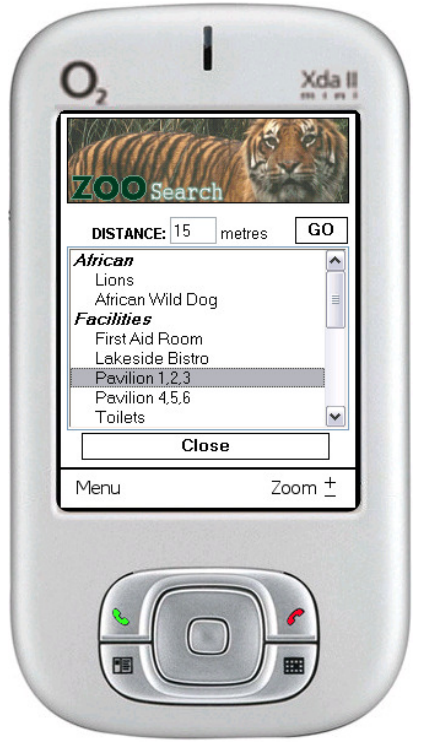

Figure 12. Search Screen [5]

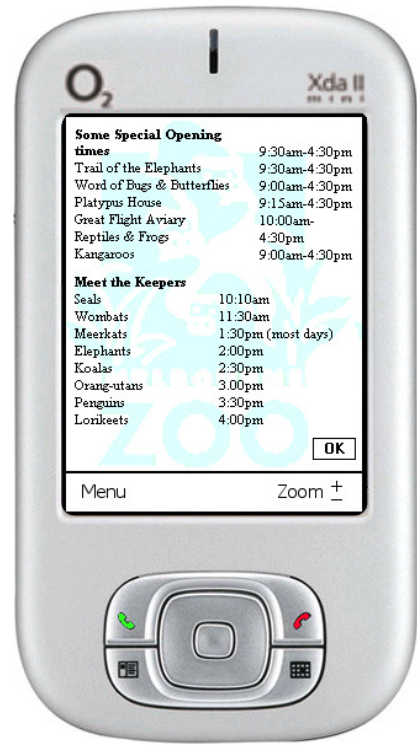

Figure 13. Events Screen [5]

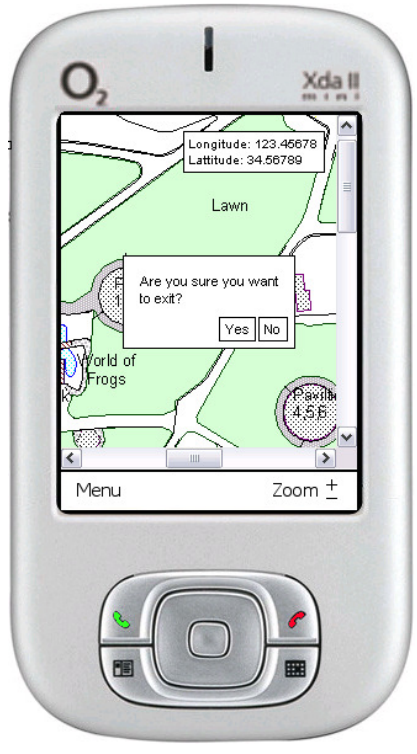

Figure 14. Exit Screen [5]

\section{Conclusions}

The ZooOz system proved to work despite minor technical problems and manages to achieve the ease of navigation around the 'Big Cats' section of the Melbourne Zoo. However, many areas within Melbourne Zoo are sheltered by trees and structures which make it hard to obtain accurate results when signals are easily obstructed by them. An ideal solution would be to use localized sensory points attached in every animal section via BlueTooth that feed the device information as the user approaches. This way the zoo has control of the signalling power as well as the content that is presented to the user.

According to Richard Langley, a GPS expert and a professor of geomatics at the University of New Brunswick, there is another GPS-like solution that is currently being worked on called "Assisted GPS" where the phone network can aid in determining an accurate location, even in areas without a clear view of the sky. The intention is to get GPS working indoors as well as it does outdoors by using antenna technology [7]. Rapid development in mobile technology in recent years has allowed GPS to work freely on mobile phones. An example of this is the Apple iPhone and the "Maps" application where you can get directions to a particular location by inputting the start and end location of your journey and track your progress via GPS [8]. 
International Journal of Distributed and Parallel Systems (IJDPS) Vol.1, No.2, November 2010

\section{ACKNOWLEDGEMENTS}

The authors would like to thank our industry partner, Rick Hammond from the Melbourne Zoo, Megan Chudleigh, Associate Professor Jordan Shan, Luke Low, Dr. Donna Dwyer and for their collaboration. Special thanks go to our students, who contributed to this project, Steven Manceski his contribution to coding and database design, Stephanie Wulf , Vanessa Jalovec and Jaesel Magallanes for the multimedia content, George Fotinos and Travis Mollica for their involvement on the business end of the project. This project was funded by the Teaching and Learning Support (TLS) grants from Victoria University.

\section{REFERENCES}

[1] Global Positioning System, http://en.wikipedia.org/wiki/Global_Positioning_System

[2] History of Mobile Phones, http://en.wikipedia.org/wiki/History_of_mobile_phones

[3] H. Shi, “An Interactive Zoo Guide:A Case Study of Collaborative Learning”, The International Journal of Multimedia \& Its Applications (IJMA), Vol. 2, No. 2, May 2010, pp. 57-67.

[4] The Interactive ZooOz Guide - Project History - Final Year Industry Project, http://thangcao.brinkster.net/zoooz.asp

[5] T. Nguyen and S. Manceski, The Interactive ZooOz Guide, 2007 Final-Year Design Project Report, School of Computer Science and Mathematics, Victoria University, Melbourne, Australia.

[6] Bluetooth Settings for Windows Mobile 5.0 Pocket PC, http://www.mypocketpcmobile.com/BluetoothSettingsonWM50/tabid/146/Default.aspx

[7] Mapping out the future of GPS, http://www.thestar.com/news/sciencetech/technology/article/813598--mapping-out-the-futureof-gps

[8] Apple - iPhone - Get directions with GPS maps and a new compass, http://www.apple.com/au/iphone/iphone-3gs/maps-compass.html

\section{Author}

Thang Nguyen obtained his Bachelor of Science in Computer Science from School of Engineering and Science, Victoria University, Melbourne, Australia in 2008. He completed the Interactive $\mathrm{ZooOz}$ Guide project under supervision of Dr. Hao Shi in 2007. He has mastered several program languages including ASP .NET, PHP, C\#, C++, PHP, Java and SQL. Thang is currently working as a Web Programmer at DMC group, Melbourne, Australia.

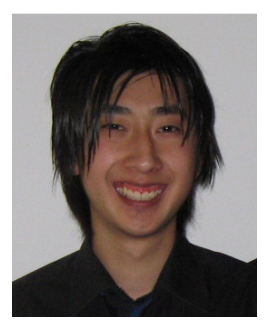

Dr. Hao Shi is an Associate Professor in the School of Engineering and Science at Victoria University, Australia. She completed her $\mathrm{PhD}$ in the area of Computer Engineering at the University of Wollongong and obtained her Bachelor of Engineering degree from Shanghai Jiao Tong University, China. She has been actively engaged in R\&D and external consultancy activities. Her research interests include p2p Networks, Location-Based Services, Web Services, Computer/Robotics Vision, eHealth, Internet and Multimedia Technologies

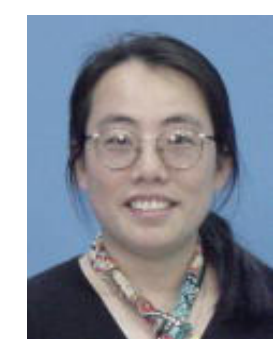

